\title{
Exchange Rates and Wages in an Integrated World
}

\section{By Prachi Mishra and Antonio Spilimbergo}

\section{Web Appendix}

\section{Wage data}

The statistics on wages are from the ILO's Key Indicators of the Labor Market (KILM). The ILO reports average earnings per worker or, in some cases, average wage rates. Some of the series cover wage earners (i.e., manual or production workers) only, while others refer to salaried employees (i.e., non-manual workers), or all employees (i.e., wage earners and salaried employees). The series cover workers of both sexes, irrespective of age..$^{38}$

The concept of earnings relates to remuneration in cash and in kind paid to employees, as a rule at regular intervals, for time worked or work done together with remuneration for time not worked, such as for annual vacation, other paid leave or holidays. In general, earnings exclude employers' contributions in respect of their employees paid to social security and pension schemes and also the benefits received by employees under these schemes. However, some countries report any such payments made. Earnings also exclude severance and termination pay. Statistics on earnings relate to employees' gross remuneration, i.e., the total before any deduction is made by the employer in respect of taxes, contributions of employees to social security and pension schemes, life insurance premiums, union dues and other obligations of employees.

Specifically, earnings include: direct wages and salaries, remuneration for time not worked (excluding severance and termination pay), bonuses and gratuities and housing and family allowances paid by the employer directly to this employee. The detailed components are as follows: (a) direct wages and salaries for time worked, or work done, cover: (i) straight time pay of time-rated workers; (ii)

\footnotetext{
38 See also Hassett and Mathur (2008), who provide details on the ILO wage data.
} 
incentive pay of time-rated workers; (iii) earnings of piece workers (excluding overtime premiums); (iv) premium pay for overtime, shift, night and holiday work; (v) commissions paid to sales and other personnel. Included are premiums for seniority and special skills, geographical zone differentials, responsibility premiums, dirt, danger and discomfort allowances, payments under guaranteed wage systems, cost-of-living allowances and other regular allowances. (b) Remuneration for time not worked comprises direct payments to employees in respect of public holidays, annual vacations and other time off with pay granted by the employer. (c) Bonuses and gratuities cover seasonal and end-of-year bonuses, additional payments in respect of vacation period (supplementary to normal pay) and profit-sharing bonuses. (ii) Statistics on earnings distinguish cash earnings from payments in kind. Wage rates: These include basic wages, cost-of-living allowances and other guaranteed and regularly paid allowances, but exclude overtime payments, bonuses and gratuities, family allowances and other social security payments made by employers. Ex gratia payments in kind, supplementary to normal wage rates, are also excluded.

The coverage of the data differs for countries because of the following reasons (1) whether the reported statistic is wages or earnings; (2) whether it covers employees, wage earners or salaried employees; and (3) whether it includes social security contributions by employer. When we studied the descriptions more closely, we found that certain countries like Chile, Turkey, Colombia, Ecuador, Kenya, Kyrgyzstan, Mexico, Malaysia, Panama and Ukraine included social security contributions by employers in the earnings data. Another difference arises because the industrial classification changed during this period. Since the beginning of the 1990s, an increasing number of countries have made a switchover in their data reporting systems for industrial statistics from Revision 2 to Revision 3 of the International Standard Classification (ISIC). We include country fixed effects to allow for all these differences in coverage in the panel regression.

\section{Data on Migration}


The main sources of the OECD migration statistics are population registers, residence or work permits, censuses and surveys. However, a wide variety of other data sources (e.g., special surveys, counts at border crossings, analysis of landing cards) are also used.

In the data, the immigrant population is usually defined in one of two ways. Some countries, including European countries, Japan, and Korea, have traditionally defined immigrants as foreign nationals living in the country whilst others, including Australia, Canada, New Zealand and the United States, define immigrants as foreign-born living in the country. This difference in definition relates in part to the nature and the history of immigration systems and legislation on citizenship and naturalization.

The foreign-born population can be viewed as representing first-generation migrants, and may consist of both foreign and nationalized citizens. The size and composition of the foreign-born population is influenced by the history of migration flows and mortality amongst the foreign-born. For example, where inflows have been declining over time, the stock of the foreign-born will tend to age and represent an increasingly established community.

On the other hand, the population of foreign nationals may represent second and higher generations as well as first generations of migrants. The characteristics of the population of foreign nationals depend on a number of factors including (i) the history of migration flows, (ii) the natural increase in the foreign population, and (iii) the naturalization rate. Higher generations of immigrants arise in situations where they retain their foreign citizenship even when native-born. The nature of legislation on citizenship and the incentives foreigners have to naturalize both play a role in determining the extent to which this occurs in practice. In some countries, such as the United States, those who are native born but who are foreign nationals are a non-existent or negligible group as legislation is such that birth within the country usually entitles individuals to citizenship. In addition to the problem of the comparability of statistics, there is the difficulty of 
the partial coverage of illegal migrants. Part of this population can be counted through censuses. The number of immigrants who entered legally but then overstay after their residence permits (or visa) have expired can be calculated from permit statistics, but without it being possible to determine what the number of these immigrants that have left the country. Regularization programs, when they exist, make it possible to account for a far from negligible fraction of illegal immigrants after the fact. In terms of measurement, this makes it possible better to evaluate the volume of the foreign population at a given time, although it is not always possible to classify these immigrants by the year when they entered the country.

\section{Derivation of labor demand}

The labor demand in equation (1) can be derived from a Cobb-Douglas production function and a standard Keynesian framework. Given the production function $Y=L^{\alpha} K^{1-\alpha}$, the demand for labor is $L^{d}=\alpha \frac{P}{w} Y$ where $\mathrm{P}$ is the price of output. In a very simple macro framework, aggregate demand is $Y^{d}=C+G+I+N X\left(\frac{e P^{*}}{P}\right)$, which can re-written as $Y^{d}=\frac{1}{1-\beta}\left[\left(\bar{C}+G+I+N X\left(\frac{e P^{*}}{P}\right)\right)\right]$ where $\beta$ is the marginal propensity to consume. $C, G$ are private and government consumption respectively. $I$ is investment demand.

Putting together aggregate demand and the production functions yields:

$L^{d}=\alpha \frac{P}{w} Y=\left(\frac{P}{w}\right) \frac{\alpha}{1-\beta}\left[\bar{C}+G+I+N X\left(\frac{e P^{*}}{P}\right)\right]$ or $L^{d}=\left(\frac{P}{w}\right) \alpha^{\prime}\left[\bar{D}+N X\left(\frac{e P^{*}}{P}\right)\right]$ which forms the basis for the labor demand presented in the text in Equation (1): $L^{d}=\left(\frac{w}{P}\right)^{-\alpha}\left[N X\left(\frac{e P^{*}}{P}\right)\right]^{\eta} \bar{D}$. 
Note that we allow for a richer specification than Cobb-Douglas production function, which imposes $\alpha=1 .{ }^{39}$

\section{Derivation of labor supply}

The labor supply presented in equation (2) can be derived from the following household utility

function $U=C-\frac{1}{\delta}\left(L^{s^{\eta}}+\sum_{i=1}^{N} \frac{1}{\phi_{i}} L_{i}^{\eta}\right)^{\frac{\delta}{\eta}}$ with $\eta<1, \delta>1$, and the constraint $L^{s}+\sum_{i=1}^{N} L_{i} \leq \bar{L}$ where $L^{s}$ is

the domestic labor supply and $L_{i}$ is the labor supply by nationals abroad in country $i$. This

functional form is an extension of the form $U=C-\frac{1}{\delta} L^{\delta}$ which is used in macro models of (labor-

closed) economies with the resulting labor supply $L^{s}=\left(\frac{w}{p}\right)^{\frac{1}{\delta-1}}$. The parameter $\frac{1}{\phi_{i}}$ measures the

disutility from working in country $i$. The maximization problem for the household is:

$\max _{L^{s} L^{*} C} U \equiv C-\frac{1}{\delta}\left(L^{s^{\eta}}+\sum_{i=1}^{N} \frac{1}{\phi_{i}} L_{i}^{\eta}\right)^{\frac{\delta}{\eta}} \quad$ s.t. $\quad P C=w L^{s}+\sum_{i=1}^{N} e_{i} w_{i} L_{i}$

Maximizing with respect to $L^{*}$ and $L_{i}$ yields: ${ }^{40}$

$L^{s}=\left(\frac{w}{P} \frac{1}{\bar{P}\left(\frac{\phi_{i} e_{i} w_{i}}{P_{i}}\right)}\right)^{\frac{1}{\delta-1}}$ where $\bar{P}\left(\frac{\phi_{i} e_{i} w_{i}}{P_{i}}\right)$ is the standard price index in CES functions

\footnotetext{
${ }^{39}$ In additional robustness checks, we include household consumption, government consumption expenditure and investment (measured by gross fixed capital formation) expenditures in all the regressions in Table 2 . The results (available upon request) are qualitatively very similar to Table 2 .

40 This is an approximation under the assumption that the number of foreign countries is large.
} 
This motivates the empirical specification used in the text.

Derivation of the relationship between wages of immigrants in the U.S. and labor market integration with the U.S.

Consider two countries, the U.S. and the origin country of immigrants $(i)$. Assume that labor is homogenous and that the labor market in the U.S. is segmented according to the nationality of immigrants.

Labor demand for immigrants from country $i$ in the U.S.

As discussed in the text, we assume that immigrant workers are imperfect substitutes for domestic workers. The resulting labor demand for immigrant workers from country $i$ is:

$L_{i}^{d, U S}=\left(\frac{w_{i}^{U S}}{P^{U S}}\right)^{-\beta} X^{d, U S}$

Where $L_{i}^{d, U S}$ is the labor demand for immigrants from country $i$ in the U.S., $w_{i}^{U S}$ is the nominal wage of immigrants from country $i$ in the U.S., $P^{U S}$ is the price index in the U.S., $X^{d, U S}$ is a composite term that captures the other factors like income in the U.S., which affect labor demand. Labor supply of immigrants from country $i$ in the U.S.

Labor supply of immigrants in the U.S. is specified as follows.

$L_{i}^{s, U S}=\left(\frac{w_{i}^{U S}}{P_{i}} * e_{i}\right)^{\delta I_{i, U S}} X^{s, i} X^{s, U S}$

Where $L_{i}^{s, U S}$ is the labor supply of immigrants in the U.S. from origin country $i ; P_{i}$ is the domestic price in origin country $i$ and $e_{i}$ is the nominal exchange rate in local currency units per US\$ in 
origin country $i{ }^{41} X^{s, U S}$ and $X^{s, i}$ are composite terms that reflect other factors respectively in the U.S. and in the origin country, affecting labor supply of immigrants in the U.S.. These capture the push and pull factors that are likely to affect labor supply of immigrants in the U.S.. The key innovation once again in the paper is to introduce $I_{i, U S}$ in the labor supply equation. We assume $\delta>0$, i.e., ceteris paribus, an increase in the real exchange rate in the origin country increases the labor supply of immigrants in the U.S. Moreover, higher is the degree of integration of the labor market with the U.S., a given increase in the real exchange rate leads to a bigger increase in labor supply. In equilibrium, assuming segmented labor markets for immigrants in the U.S., $L_{i}^{d, U S}=L_{i}^{s, U S}$

Taking logs of (A3) and simplifying:

$\ln \frac{w_{i}^{U S}}{P^{U S}}=-f\left(I_{i, U S}\right) * \ln \frac{e_{i}}{P_{i}}+x^{U S}+x^{i}$

Where $f\left(I_{i, U S}\right)=\frac{\delta I_{i, U S}}{\beta+\delta I_{i, U S}}>0 ; f^{\prime}\left(I_{i, U S}\right)>0 ; x^{U S}$ and $x^{i}$ are control variables in the U.S. and origin country $i$ respectively that affect real wages of migrants in the U.S.. Equation (A4) forms the basis of our empirical specification with real wages of immigrants in the U.S. as the dependent variable. Equation (A4) implies that the higher the degree of labor market integration of an origin country with the U.S., a given change in real exchange rate leads to a larger drop in wages.

\footnotetext{
${ }^{41}$ Note that for simplicity we are deflating wages in the U.S. by origin country price index; in other words, we assume that a migrant even if he works in the U.S. consumes his wage in the origin country. If we assume that only a share of wages earned abroad $\beta$ is spent at home and the rest is spent in the U.S., the labor supply equation can be modified as $L_{i}^{s, U S}=\left(\frac{w_{i}^{U S}}{P_{i}^{\beta}\left(P^{U S}\right)^{1-\beta}} * e_{i}\right)^{\delta I_{i, U S}} X^{s, i} X^{s, U S}$. While all results go through, we prefer to keep the simple notation in
} Equation (A2). 
Table A1. Countries in the Sample and Top Destination Countries, 1981-2005 (share of migrants in parentheses)

\begin{tabular}{|c|c|c|c|c|c|}
\hline $\begin{array}{l}\text { Origin } \\
\text { Country }\end{array}$ & First Destination & Second Destination & Third Destination & Fourth Destination & Fifth Destination \\
\hline Australia & US (46) & UK (37) & Japan (6) & Germany (4) & Ireland (2) \\
\hline Austria & Germany (61) & US (22) & Switzerland (10) & Italy (3) & Netherlands (1) \\
\hline Azerbaijan & Greece (84) & Poland (55) & Italy $(31)$ & & \\
\hline Belgium & US (23) & Netherlands (20) & Germany (18) & Luxembourg (12) & Spain (10) \\
\hline Bolivia & US (96) & Italy (3) & Sweden (2) & Netherlands $(.3)$ & \\
\hline Botswana & Netherlands (82) & Italy (18) & & & \\
\hline Brazil & Japan (49) & US (33) & Portugal (10) & Italy (4) & Spain (2) \\
\hline Bulgaria & Turkey (89) & Germany (40) & Greece (17) & Italy (12) & Czech Rep (4) \\
\hline Canada & US (89) & UK (3) & Australia (3) & Germany (1) & Greece (1) \\
\hline Chile & US (61) & Australia (20) & Sweden $(8)$ & Spain (5) & Italy (2) \\
\hline China & US (44) & Japan (17) & Canada (15) & Australia (7) & Korea (3) \\
\hline Colombia & US (89) & Spain (8) & Italy (2) & Norway (.6) & Netherlands (.3) \\
\hline Costa Rica & US (99) & Italy (.6) & Netherlands (.2) & & \\
\hline Croatia & Germany (55) & Australia (14) & Austria (13) & Switzerland (11) & Italy (4) \\
\hline Cyprus & Australia (56) & Turkey (26) & Greece (17) & Hungary $(.8)$ & Italy (.4) \\
\hline Czech Rep & US (51) & Germany (33) & Slovak Rep (6) & Italy (5) & Netherlands (2) \\
\hline Denmark & Sweden (25) & Germany (20) & US (19) & Norway (18) & Spain (6) \\
\hline Dominican Re & US (94) & Spain (4) & Italy (2) & Netherlands (.2) & Greece $(.0)$ \\
\hline Ecuador & US (79) & Spain (17) & Italy (4) & Netherlands (.1) & \\
\hline El Salvador & US (99) & Mexico (.8) & Italy (.4) & Sweden $(.1))$ & Netherlands (.0) \\
\hline Estonia & Finland (84) & Sweden (12) & Italy (2) & Poland (2.) & \\
\hline Finland & Sweden (61) & US (18) & Germany (10) & Norway (4) & Belgium (2) \\
\hline France & US (26) & Germany (14) & Belgium (14) & UK (10) & Canada (9) \\
\hline Germany & US (57) & Canada (9) & Austria (6) & Australia (6) & Switzerland (6) \\
\hline Ghana & US (65) & UK (20) & Italy (13) & Netherlands (2) & Greece $(.3)$ \\
\hline Guatemala & US (99) & Mexico (3) & Italy (.1) & Netherlands $(.0)$ & \\
\hline Hungary & US (31) & Germany (21) & Canada (18) & Australia (9) & Austria (9) \\
\hline Iceland & Denmark (40) & Sweden (28) & Norway (27) & Netherlands (3) & Luxembourg (2) \\
\hline Israel & US (89) & Italy (3) & Netherlands (2.) & Denmark (2) & Hungary (1) \\
\hline Jamaica & US (73) & Canada (20) & UK (8) & Netherlands $(.0)$ & Italy $(.0)$ \\
\hline Japan & US (82) & Germany (6) & UK (5) & Korea $(2)$ & New Zealand (1) \\
\hline Kazakhstan & Greece (98) & Poland (41) & Italy (23) & Hungary (7) & \\
\hline Kenya & US (93) & New Zealand (2) & Italy (2) & Sweden (1) & Netherlands (.8) \\
\hline Korea & US (55) & Japan (39) & Australia (3) & Germany (1) & New Zealand (1) \\
\hline Kyrgyz Rep & Greece (65) & Italy (26) & Hungary (9) & & \\
\hline Latvia & US (84) & Ireland (8) & Sweden (4) & Italy (3) & Poland (.5) \\
\hline Lithuania & US (91) & Ireland (4) & Sweden (2) & Poland (2) & Italy (2) \\
\hline Macedonia & Switzerland (30) & Germany (3) & Australia (25) & Italy (13) & Sweden (.9) \\
\hline Malaysia & Australia (49) & US (26) & UK (15) & New Zealand (7) & Japan (5) \\
\hline Mauritius & Italy (99) & Netherlands (.8) & & & \\
\hline Mexico & US (99) & Germany (.1) & Italy (.1) & Netherlands $(.0)$ & Sweden $(.0)$ \\
\hline Netherlands & Canada (19) & Germany (18) & Belgium (15) & Australia (15) & US (13) \\
\hline New Zealand & Australia (86) & UK (9) & US (4) & Ireland $(.3)$ & Netherlands (.2) \\
\hline
\end{tabular}




\begin{tabular}{llllll} 
Nicaragua & US (99.832) & Italy (.1) & Netherlands (.0) & & \\
Norway & Sweden (44) & US (22) & Denmark (17) & Germany (10.) & Netherlands (3) \\
Pakistan & US (55) & UK (20) & Germany (8) & Italy (5) & Spain (3) \\
Panama & US (99) & Italy (.5) & Greece (.2) & Netherlands (.0) & \\
Philippines & US (67) & Canada (12) & Japan (8) & Australia (6) & Italy (3) \\
Poland & US (36) & Germany (27) & Canada (15) & Australia (6) & Austria (4) \\
Portugal & France (44) & US (15) & Canada (14) & Switzerland (13) & Germany (12) \\
Romania & Italy (23) & Germany (21) & US (17) & Hungary (11) & Austria (9) \\
Russia & US (68) & Germany (19) & Finland (3) & Greece (3) & Italy (2) \\
Singapore & Australia (57) & US (36) & New Zealand (6) & Netherlands (1) & Italy (.2) \\
Slovak Rep & Czech Rep (46) & US (34) & Germany (14) & Italy (3) & Hungary (2) \\
Slovenia & Germany (46) & Austria (37) & Italy (9) & Switzerland (6) & Sweden (1) \\
South Africa & US (31) & Australia (30) & UK (23) & New Zealand (9) & Portugal (4) \\
Spain & Germany (27) & US (22) & Switzerland (17) & Belgium (10) & UK (9) \\
St Vincent Gr & Greece (92) & Netherlands (6) & Italy (1.6) & & \\
Switzerland & Germany (31) & US (28) & Italy (13) & Portugal (11) & Spain (6) \\
Thailand & US (73) & Japan (15) & Sweden (3) & New Zealand (2) & Denmark (2) \\
Trinidad Tob & US (99) & Netherlands (.1) & Italy (.0) & & \\
Turkey & Germany (70) & France (8) & Austria (.6) & Netherlands (4) & US (4.) \\
UK & Australia (37) & US (24) & Canada (20) & New Zealand (7) & Germany (4) \\
US & Canada (30) & UK (19) & Germany (14) & Australia (7) & Japan (6) \\
Ukraine & US (46) & Germany (21) & Portugal (12) & Czech Rep (11) & Italy (3) \\
Zimbabwe & UK (85) & New Zealand (12) & Greece (2) & Netherlands (.6) & Italy (.4) \\
\hline \hline
\end{tabular}

Notes. For each country, the share of migrants corresponds to the year in the period 1981-2005 with the maximum number of destination countries. Migrants in the OECD countries are defined by nationality or country of birth. 
Table A2. Emigration Rates to the OECD 1995-2005

\begin{tabular}{|c|c|c|c|}
\hline country & $\begin{array}{l}\text { Emigration rate in } \\
2005\end{array}$ & country & $\begin{array}{c}\text { Emigration rate in } \\
1995\end{array}$ \\
\hline Jamaica & 24.88 & Jamaica & 27.54 \\
\hline El Salvador & 16.64 & Portugal & 13.32 \\
\hline Trinidad Tob & 13.90 & El Salvador & 12.65 \\
\hline New Zealand & 12.53 & New Zealand & 9.59 \\
\hline Portugal & 12.12 & Trinidad Tob & 8.49 \\
\hline Mexico & 10.73 & Mexico & 7.64 \\
\hline Croatia & 8.94 & Dominican Rep & 6.94 \\
\hline Dominican Rep & 8.79 & Nicaragua & 5.50 \\
\hline Macedonia & 8.59 & UK & 5.21 \\
\hline Ecuador & 5.73 & Iceland & 4.85 \\
\hline Iceland & 5.38 & Turkey & 4.60 \\
\hline Guatemala & 4.56 & Croatia & 4.40 \\
\hline Cyprus & 4.30 & Austria & 3.56 \\
\hline Romania & 3.96 & Cyprus & 3.50 \\
\hline UK & 3.86 & Netherlands & 3.45 \\
\hline Nicaragua & 3.59 & Canada & 3.25 \\
\hline Turkey & 3.53 & Guatemala & 3.21 \\
\hline Austria & 3.43 & Poland & 3.16 \\
\hline Poland & 3.30 & Korea & 2.86 \\
\hline Netherlands & 3.26 & Finland & 2.70 \\
\hline Canada & 3.04 & Macedonia & 2.49 \\
\hline Korea & 3.00 & Hungary & 2.38 \\
\hline Panama & 2.79 & Germany & 2.25 \\
\hline Finland & 2.61 & Philippines & 2.21 \\
\hline Philippines & 2.59 & Panama & 2.15 \\
\hline Germany & 2.24 & Denmark & 1.86 \\
\hline Norway & 2.23 & Ecuador & 1.83 \\
\hline Hungary & 2.11 & Israel & 1.79 \\
\hline Slovenia & 2.06 & Spain & 1.53 \\
\hline Slovak Rep & 1.92 & Switzerland & 1.38 \\
\hline Denmark & 1.85 & Costa Rica & 1.38 \\
\hline Israel & 1.80 & Norway & 1.36 \\
\hline Spain & 1.66 & Romania & 1.13 \\
\hline Singapore & 1.56 & France & 1.12 \\
\hline Colombia & 1.56 & Slovenia & 1.07 \\
\hline Switzerland & 1.56 & Singapore & 1.07 \\
\hline Ukraine & 1.45 & Colombia & 0.96 \\
\hline Costa Rica & 1.33 & Belgium & 0.92 \\
\hline Estonia & 1.32 & Slovak Rep & 0.81 \\
\hline Belgium & 1.30 & Latvia & 0.74 \\
\hline Bulgaria & 1.26 & Estonia & 0.66 \\
\hline
\end{tabular}




\begin{tabular}{llll} 
France & 1.15 & Malaysia & 0.65 \\
Latvia & 1.01 & Bulgaria & 0.61 \\
Australia & 0.93 & Chile & 0.59 \\
Ghana & 0.83 & Australia & 0.47 \\
Malaysia & 0.79 & Mauritius & 0.46 \\
Chile & 0.75 & Japan & 0.38 \\
Mauritius & 0.75 & Bolivia & 0.35 \\
Lithuania & 0.67 & Russia & 0.34 \\
Bolivia & 0.64 & Lithuania & 0.31 \\
Russia & 0.58 & South Africa & 0.31 \\
South Africa & 0.58 & Thailand & 0.31 \\
Czech Rep & 0.58 & US & 0.27 \\
Zimbabwe & 0.52 & Ukraine & 0.20 \\
Japan & 0.46 & Brazil & 0.19 \\
Thailand & 0.44 & Czech Rep & 0.16 \\
Brazil & 0.43 & Pakistan & 0.14 \\
Pakistan & 0.29 & Ghana & 0.13 \\
China & 0.19 & China & 0.10 \\
US & 0.19 & Kenya & 0.02 \\
Kenya & 0.19 & St Vincent Gr & 0.01 \\
St Vincent Gr & 0.00 & Botswana & 0.00 \\
Botswana & 0.00 & Kyrgyz Rep & 0.00 \\
Kazakhstan & 0.00 & Kazakhstan & 0.00 \\
Kyrgyz Rep & 0.00 & Zimbabwe & 0.00 \\
& & & \\
\hline \hline & & & \\
\hline
\end{tabular}

Emigration rates are calculated by the total stock of migrants in the OECD (defined by nationality or birth) as ratio of the population in the source country. 
Table A3. Years in the Sample. 19812005

\begin{tabular}{lc}
\hline \hline Year & Number of observations \\
\hline 1981 & 22 \\
1982 & 24 \\
1983 & 22 \\
1984 & 22 \\
1985 & 20 \\
1986 & 25 \\
1987 & 24 \\
1988 & 24 \\
1989 & 25 \\
1990 & 25 \\
1991 & 27 \\
1992 & 29 \\
1993 & 29 \\
1994 & 28 \\
1995 & 29 \\
1996 & 38 \\
1997 & 48 \\
1998 & 48 \\
1999 & 49 \\
2000 & 50 \\
2001 & 39 \\
2002 & 40 \\
2003 & 26 \\
2004 & 22 \\
2005 & \\
\hline Total & \\
\hline & 240 \\
\hline
\end{tabular}


Table A4. Summary Statistics

\begin{tabular}{|c|c|c|c|c|c|}
\hline Variable & Obs & Mean & Std. Dev. & Min & $\operatorname{Max}$ \\
\hline Real wage per hour (local currency units) & 378 & 2.62 & 4.89 & 0.01 & 33.79 \\
\hline Lag migration-weighted real exchange rate (initial sample weights) & 378 & -3.02 & 2.61 & -12.30 & 3.41 \\
\hline Lag emigration rate to the OECD & 378 & 1.24 & 2.14 & 0.00 & 17.55 \\
\hline Lag exports / GDP & 378 & 26.16 & 19.80 & 4.75 & 148.25 \\
\hline Lag imports/GDP & 378 & 29.74 & 20.47 & 5.24 & 147.33 \\
\hline Lag per capita real GDP growth & 378 & 0.05 & 0.03 & -0.10 & 0.16 \\
\hline Lag unemployment rate & 378 & 8.36 & 4.62 & 0.90 & 25.20 \\
\hline Lag tax wedge & 378 & 41.78 & 13.99 & 6.93 & 82.94 \\
\hline Lag FDI/GDP & 378 & 2.87 & 3.25 & 0.00 & 22.43 \\
\hline Migration-weighted nominal wage per hour in the OECD (local currency units) & 378 & 720 & 3,777 & 0.26 & 33,577 \\
\hline Migration-weighted CPI in the OECD & 378 & 82.26 & 21.50 & 6.18 & 113.86 \\
\hline Lag real exchange rate (local currency units per US\$) & 378 & 1.24 & 3.10 & 0.00 & 30.77 \\
\hline Lag real migration-weighted exchange rate (time-varying weights) & 378 & 2.88 & 9.08 & 0.00 & 118.83 \\
\hline Lag real migration-weighted exchange rate (sample average weights) & 378 & 0.72 & 2.23 & 0.00 & 28.91 \\
\hline Lag real migration-weighted exchange rate (1995 weights) & 378 & 0.80 & 2.38 & 0.00 & 29.37 \\
\hline Lag real trade-weighted exchange rate & 378 & 5.79 & 62.82 & 0.74 & 1,173 \\
\hline Lag Remittances/GDP & 295 & 1.36 & 2.21 & 0.04 & 13.40 \\
\hline Lag stock of migrants in the OECD (in '000) & 378 & 235.22 & 477.16 & 0.08 & 5,896 \\
\hline Share of capital-intensive exports in overall (in percent) & 378 & 45.41 & 14.13 & 21.47 & 86.73 \\
\hline Share of capital-intensive imports in overall (in percent) & 378 & 43.56 & 7.04 & 31.59 & 69.52 \\
\hline Real wage from IFS (index number) & 208 & 89.70 & 28.75 & 6.45 & 274.46 \\
\hline Real wage per month; Freeman-Oostendorp (local currency units) & 151 & 2,411 & 12,725 & 4.26 & 132,243 \\
\hline Low-skill real wage per month; Freeman-Oostendorp database (local currency units) & 151 & 1,977 & 9,916 & 3.94 & 95,869 \\
\hline High-skill real wage per month; Freeman-Oostendorp database (local currency units) & 151 & 3,064 & 17,219 & 4.85 & 189,400 \\
\hline Real wage per hour of immigrants in the United States & 264 & 5.60 & 3.51 & 0.31 & 24.63 \\
\hline Lag emigration rate to the US & 264 & 1.95 & 3.59 & 0.03 & 25.52 \\
\hline
\end{tabular}




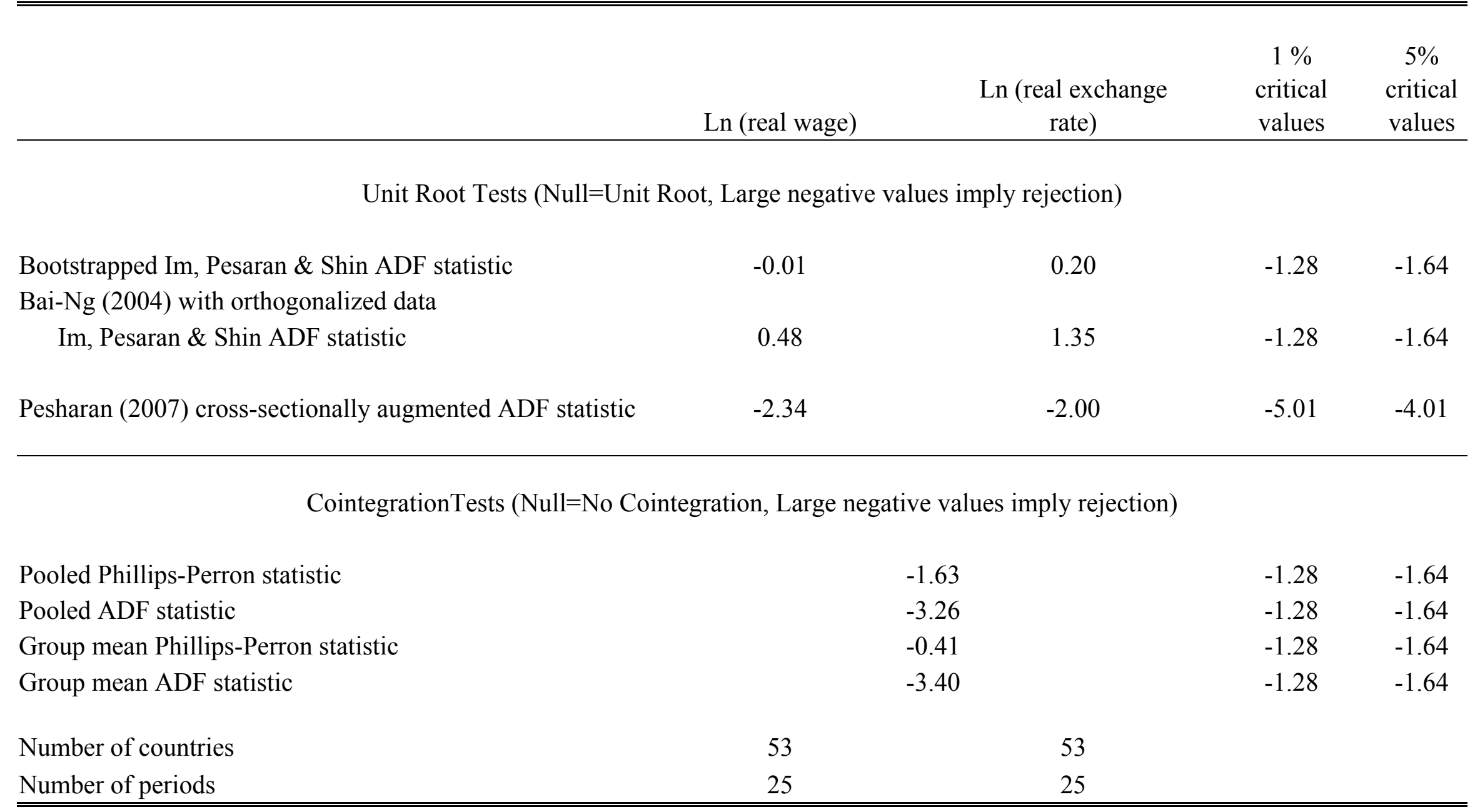

Notes. We drop countries with less than 10 years of data. The missing values for intermediate years have been interpolated to apply the unit root and cointegration tests. All reported test statistics (except for Pesharan (2007) test statitic) are distributed $\mathrm{N}(0,1)$ under null of unit root or no cointegration). The reported values of test statistics shown in the table are calculated assuming 3 lags. The values are similar if we use 4,5 or 6 lags. 
Table A6. Effect of Exchange Rates on Wages-Interaction of Exchange Rates with LaborDD

\begin{tabular}{|c|c|c|c|c|}
\hline \multicolumn{5}{|c|}{ Dependent variable: $\ln ($ real wage $)$} \\
\hline & {$[1]$} & {$[2]$} & [3] & [4] \\
\hline Ln migration-weighted real exchange rate $t_{t-1}$ & $\begin{array}{l}-0.079 \\
(0.284\end{array}$ & $\begin{array}{c}0.385 \\
(0.365)\end{array}$ & $\begin{array}{c}0.156 \\
(0.322)\end{array}$ & $\begin{array}{c}0.203 \\
(0.307)\end{array}$ \\
\hline $\begin{array}{l}\text { Ln migration-weighted real exchange rate } \\
\text { rate }_{t-1} * \text { In emigration }\end{array}$ & $\begin{array}{l}0.044 * * \\
(0.021)\end{array}$ & $\begin{array}{l}0.051 * * \\
(0.023)\end{array}$ & $\begin{array}{l}0.053 * * \\
(0.022)\end{array}$ & $\begin{array}{l}0.051 * * \\
(0.022)\end{array}$ \\
\hline Ln emigration rate $t_{t-1}$ & $\begin{array}{c}0.128 * * * \\
(0.040)\end{array}$ & $\begin{array}{c}0.140 * * * \\
(0.044)\end{array}$ & $\begin{array}{c}0.144 * * * \\
(0.043)\end{array}$ & $\begin{array}{c}0.131^{* * *} \\
(0.044)\end{array}$ \\
\hline $\operatorname{Ln}(\text { exports/GDP) })_{\mathrm{t}-1}$ & $\begin{array}{l}0.552 * \\
(0.291)\end{array}$ & $\begin{array}{c}0.225 \\
(0.278)\end{array}$ & $\begin{array}{c}0.846 * * * \\
(0.272)\end{array}$ & $\begin{array}{c}0.864 * * * \\
(0.247)\end{array}$ \\
\hline Ln (imports/GDP) $)_{\mathrm{t}-1}$ & $\begin{array}{c}-0.478^{* *} \\
(0.215)\end{array}$ & $\begin{array}{l}-0.405^{*} \\
(0.236)\end{array}$ & $\begin{array}{c}-0.922 * * * \\
(0.262)\end{array}$ & $\begin{array}{c}-1.055^{* * *} \\
(0.282)\end{array}$ \\
\hline Growth in real GDP per capita $a_{t-1}$ & $\begin{array}{l}-0.294 \\
(0.958)\end{array}$ & $\begin{array}{l}-0.244 \\
(0.947)\end{array}$ & $\begin{array}{c}-0.5 \\
(0.995)\end{array}$ & $\begin{array}{l}-0.812 \\
(1.230)\end{array}$ \\
\hline Ln unemployment rate $\mathrm{t}_{-1}$ & $\begin{array}{c}-0.141^{*} \\
(0.084)\end{array}$ & $\begin{array}{l}-0.141 \\
(0.086)\end{array}$ & $\begin{array}{l}-0.143^{*} \\
(0.082)\end{array}$ & $\begin{array}{c}-0.408^{* * *} \\
(0.131)\end{array}$ \\
\hline Ln tax wedge $\mathrm{t}_{\mathrm{t}-1}$ & $\begin{array}{c}-0.268^{* *} \\
(0.122)\end{array}$ & $\begin{array}{c}-0.257^{* *} \\
(0.122)\end{array}$ & $\begin{array}{c}-0.251^{* *} \\
(0.123)\end{array}$ & $\begin{array}{c}0.118 \\
(0.214)\end{array}$ \\
\hline $\operatorname{Ln}(\mathrm{FDI} / \mathrm{GDP})_{\mathrm{t}-1}$ & $\begin{array}{c}0.04 \\
(0.028)\end{array}$ & $\begin{array}{c}0.026 \\
(0.027)\end{array}$ & $\begin{array}{c}0.033 \\
(0.028)\end{array}$ & $\begin{array}{l}-0.065^{*} \\
(0.039)\end{array}$ \\
\hline Ln migration-weighted OECD wage $\mathrm{t}_{t-1}$ & $\begin{array}{c}0.056 \\
(0.081)\end{array}$ & $\begin{array}{c}0.066 \\
(0.082)\end{array}$ & $\begin{array}{c}0.07 \\
(0.081)\end{array}$ & $\begin{array}{l}-0.042 \\
(0.080)\end{array}$ \\
\hline Ln migration-weighted OECD price ${ }_{t-1}$ & $\begin{array}{l}-0.115 \\
(0.073)\end{array}$ & $\begin{array}{l}-0.088 \\
(0.072)\end{array}$ & $\begin{array}{c}-0.127^{*} \\
(0.077)\end{array}$ & $\begin{array}{c}0.071 \\
(0.074)\end{array}$ \\
\hline $\operatorname{Ln}(\text { exports/GDP) })_{t-1} * \operatorname{Ln}$ migration-weighted real exchange rate $t_{t-1}$ & $\begin{array}{c}0.149 * * \\
(0.068)\end{array}$ & & $\begin{array}{l}0.227 * * * \\
(0.087)\end{array}$ & $\begin{array}{c}0.286^{* * *} \\
(0.073)\end{array}$ \\
\hline Ln (imports/GDP) $)_{t-1} *$ Ln migration-weighted real exchange rate $t_{t-1}$ & & $\begin{array}{l}-0.014 \\
(0.090)\end{array}$ & $\begin{array}{c}-0.18 \\
(0.114)\end{array}$ & $\begin{array}{c}-0.239 * * \\
(0.097)\end{array}$ \\
\hline $\begin{array}{l}\text { Growth in real GDP per capita } \mathrm{t}_{\mathrm{t}-1} * \mathrm{Ln} \text { migration-weighted real } \\
\text { exchange rate } \mathrm{t}_{\mathrm{t}-1}\end{array}$ & & & & $\begin{array}{l}-0.001 \\
(0.236)\end{array}$ \\
\hline $\begin{array}{l}\text { Ln unemployment rate } \mathrm{t}_{-1} * \text { Ln migration-weighted real exchange } \\
\text { rate }_{t-1}\end{array}$ & & & & $\begin{array}{c}-0.106^{* * *} \\
(0.032)\end{array}$ \\
\hline Ln tax wedge $e_{t-1} *$ Ln migration-weighted real exchange rate $e_{t-1}$ & & & & $\begin{array}{c}0.136^{* *} \\
(0.063)\end{array}$ \\
\hline $\operatorname{Ln}(\mathrm{FDI} / \mathrm{GDP})_{\mathrm{t}-1} * \mathrm{Ln}$ migration-weighted real exchange rate $\mathrm{t}_{\mathrm{t}-1}$ & & & & $\begin{array}{c}-0.033 * * * \\
(0.010)\end{array}$ \\
\hline $\begin{array}{l}\text { Ln migration-weighted OECD wage } \mathrm{t}_{-1} * \mathrm{Ln} \text { migration-weighted } \\
\text { real exchange rate } \mathrm{t}_{\mathrm{t}-1}\end{array}$ & & & & $\begin{array}{c}-0.064 * * \\
(0.030)\end{array}$ \\
\hline $\begin{array}{l}\text { Ln migration-weighted OECD price } t_{t-1} * \text { Ln migration-weighted } \\
\text { real exchange rate } t_{t-1}\end{array}$ & & & & $\begin{array}{l}0.039^{*} \\
(0.020)\end{array}$ \\
\hline
\end{tabular}




\begin{tabular}{lcccc} 
Country fixed effects & Y & Y & Y & Y \\
Year fixed effects & Y & Y & Y & Y \\
\hline Observations & 378 & 378 & 378 & 378 \\
Number of countries & 44 & 44 & 44 & 44 \\
\hline \hline
\end{tabular}

Notes. * significant at 10\%; ${ }^{* *}$ significant at $5 \%$, *** significant at $1 \%$. Robust standard errors in parentheses.

All variables refer to the origin country of migrants except wages and prices in OECD. 
Table A7. Effect of Exchange Rates on Wages-Interaction With Labor Market Integration: Consistent Sample

\begin{tabular}{|c|c|c|c|c|c|c|}
\hline \multicolumn{7}{|c|}{ Dependent variable: $\ln ($ real wage $)$} \\
\hline & {$[1]$} & {$[2]$} & [3] & {$[4]$} & {$[5]$} & {$[6]$} \\
\hline \multirow{2}{*}{ Ln migration-weighted real exchange rate ${ }_{t-1}$} & $0.360^{*}$ & $0.362 *$ & 0.346 & 0.346 & 0.349 & 0.35 \\
\hline & $(0.216)$ & $(0.217)$ & $(0.216)$ & $(0.216)$ & $(0.215)$ & $(0.215)$ \\
\hline \multirow[t]{2}{*}{$\begin{array}{l}\text { Ln migration-weighted real exchange rate } \text { t-1 } * \text { Ln } \\
\text { emigration rate }_{t-1}\end{array}$} & $0.046 * *$ & $0.046 * *$ & $0.047 * *$ & $0.050 * *$ & $0.050 * *$ & $0.050 * *$ \\
\hline & $(\mathbf{0 . 0 2 0})$ & $(\mathbf{0 . 0 2 0})$ & $(0.021)$ & $(0.021)$ & $(\mathbf{0 . 0 2 0})$ & $(0.021)$ \\
\hline \multirow[t]{2}{*}{ Ln emigration rate ${ }_{t-1}$} & $0.124 * * *$ & $0.123 * * *$ & $0.126^{* * *}$ & $0.139 * * *$ & $0.138 * * *$ & $0.139 * * *$ \\
\hline & $(0.040)$ & $(0.040)$ & $(0.041)$ & $(0.040)$ & $(0.040)$ & $(0.041)$ \\
\hline \multirow[t]{2}{*}{$\operatorname{Ln}(\text { exports/GDP })_{\mathrm{t}-1}$} & 0.094 & 0.094 & 0.204 & 0.191 & 0.194 & 0.215 \\
\hline & $(0.256)$ & $(0.257)$ & $(0.304)$ & $(0.304)$ & $(0.303)$ & $(0.311)$ \\
\hline \multirow[t]{2}{*}{ Ln $(\text { imports/GDP) })_{\mathrm{t}-1}$} & $-0.402 *$ & $-0.411^{*}$ & $-0.489 *$ & -0.394 & -0.393 & -0.374 \\
\hline & $(0.239)$ & $(0.225)$ & $(0.249)$ & $(0.264)$ & $(0.263)$ & $(0.253)$ \\
\hline \multirow[t]{2}{*}{ Growth in real GDP per capita $\mathrm{t}_{\mathrm{t}-1}$} & & 0.114 & -0.009 & -0.143 & -0.207 & -0.23 \\
\hline & & $(0.891)$ & $(0.901)$ & $(0.913)$ & $(0.904)$ & $(0.938)$ \\
\hline \multirow[t]{2}{*}{ Ln unemployment rate $_{t-1}$} & & & $-0.146^{*}$ & -0.134 & $-0.137^{*}$ & -0.141 \\
\hline & & & $(0.083)$ & $(0.083)$ & $(0.083)$ & $(0.086)$ \\
\hline \multirow[t]{2}{*}{ Ln tax wedge $e_{t-1}$} & & & & $-0.268 * *$ & $-0.262 * *$ & $-0.259 * *$ \\
\hline & & & & $(0.119)$ & $(0.119)$ & $(0.122)$ \\
\hline \multirow[t]{2}{*}{$\operatorname{Ln}(\mathrm{FDI} / \mathrm{GDP})_{\mathrm{t}-1}$} & & & & & 0.029 & 0.027 \\
\hline & & & & & $(0.027)$ & $(0.028)$ \\
\hline \multirow[t]{2}{*}{ Ln average OECD wage t- $_{-1}$} & & & & & & 0.065 \\
\hline & & & & & & $(0.082)$ \\
\hline \multirow[t]{2}{*}{ Ln average OECD price $_{t-1}$} & & & & & & -0.088 \\
\hline & & & & & & $(0.072)$ \\
\hline Country fixed effects & Y & Y & $\mathrm{Y}$ & Y & Y & Y \\
\hline Year fixed effects & $\mathrm{Y}$ & $\mathrm{Y}$ & $\mathrm{Y}$ & $\mathrm{Y}$ & $\mathrm{Y}$ & $\mathrm{Y}$ \\
\hline Observations & 378 & 378 & 378 & 378 & 378 & 378 \\
\hline Number of countries & 44 & 44 & 44 & 44 & 44 & 44 \\
\hline
\end{tabular}

Notes. * significant at 10\%; ** significant at 5\%,*** significant at $1 \%$. Robust standard errors in parentheses. All variables refer to the origin country of

migrants except wages and prices in OECD. 
Table A8. Effect of Exchange Rates on Wages-Interactions-Developing Countries:

Consistent Sample

Dependent variable: $\ln ($ real wage)

[1]

[2]

[3]

[4]

Ln migration-weighted real exchange rate $_{t-1}$
0.186
0.186
0.179
0.18
(0.203)
$(0.204)$
(0.202)
$(0.202)$

$\begin{array}{cccc}\mathbf{0 . 0 2 2} * * & \mathbf{0 . 0 2 2} * * & 0.027 * * * & 0.027 * * * \\ (0.010) & (0.010) & (0.010) & (0.010)\end{array}$

Ln migration-weighted real exchange rate $_{t-1}$ * Ln emigration rate $_{t-1}$

Ln migration-weighted real exchange rate t- $1^{*} \mathrm{Ln}$ emigration rate $\mathrm{t}_{\mathrm{t}-1} *$ developing

$0.051^{* *} \quad 0.051^{* *} \quad 0.050^{* *} \quad 0.050^{* *}$

$(0.025) \quad(0.025) \quad(0.025)$

(0.025)

Ln emigration rate t-1 $_{1}$

$\begin{array}{llll}0.131 * * * & 0.131 * * * & 0.147 * * * & 0.146 * * *\end{array}$

Ln (exports/GDP) $)_{\mathrm{t}-1}$

$\begin{array}{llll}(0.037) & (0.037) & (0.038) & (0.039)\end{array}$

Ln (imports/GDP) $)_{\mathrm{t}-1}$

0.238

0.237

0.323

0.339

(0.267)

$(0.269)$

(0.314)

(0.322)

$-0.425^{*}$

$-0.427^{*}$

$-0.397$

$-0.377$

(0.252)

(0.238)

(0.273)

(0.263)

Dummy for crisis $\mathrm{t}_{\mathrm{t}-1}$

$0.022 \quad-0.292 \quad-0.286$

$\begin{array}{lll}(0.887) & (0.894) \quad(0.925)\end{array}$

Ln unemployment rate $_{t-1}$

$-0.123-0.123$

(0.079) (0.082)

Ln tax wedge $\mathrm{e}_{\mathrm{t}-1}$ $-0.274 * *-0.269 * *$

(0.116) (0.119)

$\operatorname{Ln}(\text { FDI/GDP })_{\mathrm{t}-1}$ $0.03 \quad 0.027$

(0.026) (0.027)

Ln average OECD wage $_{t-1}$ 0.066 (0.080)

Ln average OECD price t-1 $_{1}$ $-0.062$ (0.069)

Country fixed effects

Year fixed effects

Observations

\begin{tabular}{cccc}
$\mathrm{Y}$ & $\mathrm{Y}$ & $\mathrm{Y}$ & $\mathrm{Y}$ \\
$\mathrm{Y}$ & $\mathrm{Y}$ & $\mathrm{Y}$ & $\mathrm{Y}$ \\
\hline 378 & 378 & 378 & 378 \\
44 & 44 & 44 & 44 \\
\hline
\end{tabular}

Number of countries

Notes. significant at 10\%; ** significant at 5\%, *** significant at 1\%. Robust standard errors in parentheses. All variables refer to the origin country of migrants except wages and prices in OECD. 
Table A9. Effect of Exchange Rates on Wages-Interaction With Labor Market Integration: Differential Effect Across Regions

\begin{tabular}{|c|c|c|c|c|}
\hline \multicolumn{5}{|c|}{ Dependent variable: $\ln ($ real wage) } \\
\hline & $\begin{array}{c}\text { Asia } \\
{[1]}\end{array}$ & $\begin{array}{c}\text { Europe } \\
{[2]}\end{array}$ & $\begin{array}{c}\text { America } \\
{[3]}\end{array}$ & $\begin{array}{c}\text { Africa/ME } \\
{[4]}\end{array}$ \\
\hline Ln migration-weighted real exchange rate t- $1_{1}$ & $\begin{array}{c}0.485 * * * \\
(0.102)\end{array}$ & $\begin{array}{l}-0.163 \\
(0.335)\end{array}$ & $\begin{array}{c}0.72 \\
(0.484)\end{array}$ & $\begin{array}{c}2.444 * * * \\
(0.624)\end{array}$ \\
\hline $\begin{array}{l}\text { Ln migration-weighted real exchange rate } \\
\text { emigration } * \text { In } \\
\text { at-1 }\end{array}$ & $\begin{array}{l}0.059 * * \\
(0.022)\end{array}$ & $\begin{array}{l}0.183 * \\
(0.099)\end{array}$ & $\begin{array}{l}\mathbf{0 . 0 5 5}+ \\
(\mathbf{0 . 0 3 6 )}\end{array}$ & $\begin{array}{l}0.223 * * * \\
(\mathbf{0 . 0 5 8})\end{array}$ \\
\hline Ln emigration rate t-1 $_{1}$ & $\begin{array}{c}0.321 * * * \\
(0.071)\end{array}$ & $\begin{array}{c}0.037 \\
(0.171)\end{array}$ & $\begin{array}{l}0.160 * \\
(0.088)\end{array}$ & $\begin{array}{c}1.089 * * * \\
(0.338)\end{array}$ \\
\hline $\operatorname{Ln}(\text { exports/GDP })_{\mathrm{t}-1}$ & $\begin{array}{l}-0.393 \\
(0.233)\end{array}$ & $\begin{array}{c}1.889 * * * \\
(0.506)\end{array}$ & $\begin{array}{c}-0.940 * \\
(0.500)\end{array}$ & $\begin{array}{c}-1.007^{* *} \\
(0.356)\end{array}$ \\
\hline Ln (imports/GDP) $)_{\mathrm{t}-1}$ & $\begin{array}{c}0.112 \\
(0.228)\end{array}$ & $\begin{array}{c}-1.542 * * * \\
(0.477)\end{array}$ & $\begin{array}{c}0.565 \\
(0.609)\end{array}$ & $\begin{array}{c}-0.520 * \\
(0.240)\end{array}$ \\
\hline Growth in real GDP per capita $\mathrm{t}_{-1}$ & $\begin{array}{l}-0.744 \\
(0.679)\end{array}$ & $\begin{array}{c}0.53 \\
(1.503)\end{array}$ & $\begin{array}{l}-1.065 \\
(2.420)\end{array}$ & \\
\hline Ln unemployment rate $_{t-1}$ & $\begin{array}{c}0.006 \\
(0.079)\end{array}$ & $\begin{array}{c}-0.377 * * * \\
(0.135)\end{array}$ & $\begin{array}{c}0.504 \\
(0.331)\end{array}$ & \\
\hline Ln tax wedge $\mathrm{e}_{\mathrm{t}-1}$ & $\begin{array}{l}-0.205 \\
(0.283)\end{array}$ & $\begin{array}{c}-0.23 \\
(0.187)\end{array}$ & $\begin{array}{l}-0.003 \\
(0.430)\end{array}$ & \\
\hline $\operatorname{Ln}(\mathrm{FDI} / \mathrm{GDP})_{\mathrm{t}-1}$ & $\begin{array}{c}0.001 \\
(0.019)\end{array}$ & $\begin{array}{c}0.018 \\
(0.052)\end{array}$ & $\begin{array}{c}0.261 \\
(0.168)\end{array}$ & \\
\hline Ln migration-weighted OECD wage $_{t-1}$ & $\begin{array}{c}0.007 \\
(0.024)\end{array}$ & $\begin{array}{c}0.146 \\
(0.104)\end{array}$ & $\begin{array}{c}-0.409 * * \\
(0.197)\end{array}$ & \\
\hline Ln migration-weighted OECD price ${ }_{t-1}$ & $\begin{array}{l}-0.039 \\
(0.063)\end{array}$ & $\begin{array}{l}-0.058 \\
(0.106)\end{array}$ & $\begin{array}{c}0.1 \\
(0.210)\end{array}$ & \\
\hline Country fixed effects & $\mathrm{Y}$ & & & \\
\hline Year fixed effects & Y & & & \\
\hline Observations & 62 & 207 & 95 & 35 \\
\hline Number of countries & 7 & 23 & 11 & 7 \\
\hline
\end{tabular}

Notes. ${ }^{+}$significant at 15\%; * significant at 10\%; ** significant at 5\%, *** significant at 1\%. Robust standard errors in parentheses. All variables refer to the origin country of migrants except wages and prices in OECD. 
Table A10. Effect of Exchange Rates on Wages-Interaction With Labor Market Integration -- Drop Outliers

Dependent variable: $\ln ($ real wage)

Drop countries which

Drop Mexico and Philipines send 90\% of migrants

to US

[1]

[2]

\begin{tabular}{|c|c|c|}
\hline Ln migration-weighted real exchange rate $t_{t-1}$ & $\begin{array}{c}0.314 \\
(0.238)\end{array}$ & $\begin{array}{c}0.183 \\
(0.227)\end{array}$ \\
\hline $\begin{array}{l}\text { Ln migration-weighted real exchange } \\
\text { rate }_{t-1} * \text { In emigration rate } \\
t-1\end{array}$ & $\begin{array}{c}\mathbf{0 . 0 4 3}^{* *} \\
(0.020)\end{array}$ & $\begin{array}{c}\mathbf{0 . 0 2 2}^{+} \\
(0.014)\end{array}$ \\
\hline Ln emigration rate ${ }_{t-1}$ & $\begin{array}{l}0.088^{*} \\
(0.050)\end{array}$ & $\begin{array}{l}-0.026 \\
(0.069)\end{array}$ \\
\hline $\operatorname{Ln}(\text { exports/GDP) })_{\mathrm{t}-1}$ & $\begin{array}{c}0.352 \\
(0.363)\end{array}$ & $\begin{array}{c}0.553 \\
(0.411)\end{array}$ \\
\hline Ln (imports/GDP) $)_{t-1}$ & $\begin{array}{l}-0.382 \\
(0.296)\end{array}$ & $\begin{array}{l}-0.301 \\
(0.288)\end{array}$ \\
\hline Growth in real GDP per capita $a_{t-1}$ & $\begin{array}{l}-0.229 \\
(0.989)\end{array}$ & $\begin{array}{l}-0.252 \\
(0.953)\end{array}$ \\
\hline Ln unemployment rate $\mathrm{t}_{-1}$ & $\begin{array}{l}-0.150^{*} \\
(0.090)\end{array}$ & $\begin{array}{l}-0.174^{*} \\
(0.101)\end{array}$ \\
\hline Ln tax wedge $\mathrm{t}_{\mathrm{t}-1}$ & $\begin{array}{c}-0.360 * * * \\
(0.120)\end{array}$ & $\begin{array}{c}-0.282^{* *} \\
(0.120)\end{array}$ \\
\hline $\operatorname{Ln}(\mathrm{FDI} / \mathrm{GDP})_{\mathrm{t}-1}$ & $\begin{array}{c}0.026 \\
(0.030)\end{array}$ & $\begin{array}{c}0.031 \\
(0.029)\end{array}$ \\
\hline Ln migration-weighted OECD wage $\mathrm{e}_{t-1}$ & $\begin{array}{c}0.067 \\
(0.082)\end{array}$ & $\begin{array}{c}0.079 \\
(0.098)\end{array}$ \\
\hline Ln migration-weighted OECD price $_{t-1}$ & $\begin{array}{l}-0.088 \\
(0.074)\end{array}$ & $\begin{array}{l}-0.105 \\
(0.085)\end{array}$ \\
\hline Country fixed effects & Y & $\mathrm{Y}$ \\
\hline Year fixed effects & Y & Y \\
\hline Observations & 359 & 345 \\
\hline Number of countries & 42 & 38 \\
\hline
\end{tabular}

Notes. ${ }^{+}$significant at $15 \%$; $*$ significant at $10 \%$; ** significant at $5 \%$, *** significant at $1 \%$. Robust standard errors in parentheses. All variables refer to the origin country of migrants except wages and prices in OECD. 
Table A11. Effect of Exchange Rates on Wages-Interaction With Labor Market Integration: Split Samples by Trend Growth

Dependent variable: $\ln ($ real wage $)$

\begin{tabular}{|c|c|c|c|c|}
\hline & $\begin{array}{c}\text { High } \\
\text { growth } \\
{[1]}\end{array}$ & $\begin{array}{c}\text { Low } \\
\text { growth } \\
{[2]}\end{array}$ & $\begin{array}{c}\text { High } \\
\text { growth } \\
{[3]}\end{array}$ & $\begin{array}{c}\text { Low } \\
\text { growth } \\
{[4]}\end{array}$ \\
\hline Ln migration-weighted real exchange rate ${ }_{t-1}$ & $\begin{array}{l}-1.159 * * \\
(0.530)\end{array}$ & $\begin{array}{l}0.318 * \\
(0.190)\end{array}$ & $\begin{array}{c}0.426 \\
(0.359)\end{array}$ & $\begin{array}{l}0.338 * \\
(0.189)\end{array}$ \\
\hline $\begin{array}{l}\text { Ln migration-weighted real exchange } \text { rate }_{t-1} \\
* \text { In emigration rate } \\
t-1\end{array}$ & $-0.157 * * *$ & $0.043 *$ & 0.067 & $0.036 *$ \\
\hline & $(\mathbf{0 . 0 5 3 )}$ & $(0.022)$ & $(0.080)$ & $(0.020)$ \\
\hline Ln emigration rate t 1 & $\begin{array}{c}-0.275 * * \\
(0.112)\end{array}$ & $\begin{array}{c}0.026 \\
(0.104)\end{array}$ & $\begin{array}{c}0.187 \\
(0.149)\end{array}$ & $\begin{array}{c}0.061 \\
(0.123)\end{array}$ \\
\hline $\operatorname{Ln}(\text { exports/GDP })_{\mathrm{t}-1}$ & $\begin{array}{l}-0.093 \\
(0.559)\end{array}$ & $\begin{array}{c}0.14 \\
(0.261)\end{array}$ & $\begin{array}{c}0.373 \\
(0.493)\end{array}$ & $\begin{array}{c}0.008 \\
(0.247)\end{array}$ \\
\hline Ln (imports/GDP) $)_{\mathrm{t}-1}$ & $\begin{array}{l}1.359 \\
(0.86)\end{array}$ & $\begin{array}{l}-0.261 \\
(0.273)\end{array}$ & $\begin{array}{l}-0.494 \\
(0.659)\end{array}$ & $\begin{array}{l}-0.118 \\
(0.235)\end{array}$ \\
\hline Growth in real GDP per capita ${ }_{t-1}$ & $\begin{array}{l}-2.236 \\
(2.107)\end{array}$ & $\begin{array}{c}0.089 \\
(0.867)\end{array}$ & $\begin{array}{l}-1.062 \\
(1.705)\end{array}$ & $\begin{array}{c}0.754 \\
(0.590)\end{array}$ \\
\hline Ln unemployment rate t-1 & $\begin{array}{c}0.007 \\
(0.413)\end{array}$ & $\begin{array}{l}-0.108 \\
(0.084)\end{array}$ & $\begin{array}{l}-0.349 \\
(0.217)\end{array}$ & $\begin{array}{l}-0.018 \\
(0.063)\end{array}$ \\
\hline Ln tax wedge $e_{t-1}$ & $\begin{array}{c}0.175 \\
(0.456)\end{array}$ & $\begin{array}{l}-0.235 \\
(0.155)\end{array}$ & $\begin{array}{l}-0.145 \\
(0.264)\end{array}$ & $\begin{array}{c}-0.399 * * * \\
(0.127)\end{array}$ \\
\hline $\operatorname{Ln}(\mathrm{FDI} / \mathrm{GDP})_{\mathrm{t}-1}$ & $\begin{array}{c}-0.134 \\
-0.14\end{array}$ & $\begin{array}{c}0.036 \\
(0.027)\end{array}$ & $\begin{array}{c}0.018 \\
(0.086)\end{array}$ & $\begin{array}{c}0.045^{*} \\
(0.023)\end{array}$ \\
\hline Ln migration-weighted OECD wage t -1 & $\begin{array}{l}-0.112 \\
-0.186\end{array}$ & $\begin{array}{l}0.156^{*} \\
-0.085\end{array}$ & $\begin{array}{c}0.018 \\
(0.119)\end{array}$ & $\begin{array}{c}0.162 * * * \\
(0.053)\end{array}$ \\
\hline Ln migration-weighted OECD price t-1 & $\begin{array}{l}-0.038 \\
-0.143\end{array}$ & $\begin{array}{c}-0.214^{* *} \\
-0.098\end{array}$ & $\begin{array}{c}-0.014 \\
(0.183)\end{array}$ & $\begin{array}{l}-0.126^{*} \\
(0.072)\end{array}$ \\
\hline Country fixed effects & $\mathrm{Y}$ & $\mathrm{Y}$ & $\mathrm{Y}$ & $\mathrm{Y}$ \\
\hline Year fixed effects & Y & Y & Y & $\mathrm{Y}$ \\
\hline Observations & 90 & 285 & 183 & 192 \\
\hline Number of countries & 14 & 29 & 26 & 17 \\
\hline
\end{tabular}

The regression sample corresponding to Table 2, column [6] are split by trend growth. In the first two columns, they are defined by those above and below the 75th percentile; in the last two columns, countries with high and low trend growth are defined by those above and below the median. Notes. ${ }^{*}$ significant at $10 \%$; ${ }^{*}$ significant at $5 \%$, ${ }^{* * *}$ significant at $1 \%$. Robust standard errors in parentheses. All variables refer to the origin country of migrants except wages and prices in OECD. 
Table A12. Effect of Exchange Rates on Wages-Interaction With Labor Market Integration - Control for Composition of Trade

\begin{tabular}{|c|c|c|c|c|}
\hline \multicolumn{5}{|c|}{ Dependent variable: $\ln ($ real wage $)$} \\
\hline & {$[1]$} & {$[2]$} & [3] & [4] \\
\hline Ln migration-weighted real exchange rate $_{t-1}$ & $\begin{array}{c}0.324 * * * \\
(0.112)\end{array}$ & $\begin{array}{c}0.338 \\
(0.212)\end{array}$ & $\begin{array}{l}0.361 * * * \\
(0.122)\end{array}$ & $\begin{array}{c}0.327 \\
(0.214)\end{array}$ \\
\hline \multirow[t]{2}{*}{$\begin{array}{l}\text { Ln migration-weighted real exchange } \text { rate }_{t-1} * \text { Ln } \\
\text { emigration rate } \\
t-1\end{array}$} & $0.012 * *$ & $0.046 * *$ & $0.015 * *$ & $0.048 * *$ \\
\hline & $(0.005)$ & $(0.021)$ & $(0.006)$ & $(\mathbf{0 . 0 2 0})$ \\
\hline \multirow{2}{*}{ Ln emigration rate $_{t-1}$} & 0.022 & $0.118 * *$ & $0.050 * * *$ & $0.124 * * *$ \\
\hline & $(0.019)$ & $(0.049)$ & $(0.019)$ & $(0.044)$ \\
\hline \multirow[t]{2}{*}{$\operatorname{Ln}(\text { exports/GDP })_{\mathrm{t}-1}$} & -1.414 & -0.35 & 0.07 & 0.275 \\
\hline & $(1.543)$ & $(3.045)$ & $(0.132)$ & $(0.327)$ \\
\hline \multirow[t]{2}{*}{ Ln (imports/GDP) $)_{\mathrm{t}-1}$} & $-5.490 * * *$ & $-3.833 * *$ & $-0.941 * *$ & $-1.359 *$ \\
\hline & $(1.617)$ & $(1.724)$ & $(0.368)$ & $(0.731)$ \\
\hline \multirow{2}{*}{ Growth in real GDP per capita $\mathrm{t}_{-1}$} & & -0.134 & & 0.118 \\
\hline & & $(0.896)$ & & $(0.818)$ \\
\hline \multirow[t]{2}{*}{ Ln unemployment rate ${ }_{t-1}$} & & $-0.146^{*}$ & & -0.102 \\
\hline & & $(0.086)$ & & $(0.083)$ \\
\hline \multirow[t]{2}{*}{ Ln tax wedge $e_{t-1}$} & & $-0.316 * * *$ & & $-0.226^{*}$ \\
\hline & & $(0.115)$ & & $(0.127)$ \\
\hline \multirow[t]{2}{*}{$\operatorname{Ln}(\mathrm{FDI} / \mathrm{GDP})_{\mathrm{t}-1}$} & & 0.033 & & 0.034 \\
\hline & & $(0.029)$ & & $(0.029)$ \\
\hline \multirow[t]{2}{*}{ Ln migration-weighted OECD wage $_{t-1}$} & & 0.057 & & 0.041 \\
\hline & & $(0.081)$ & & $(0.084)$ \\
\hline \multirow{2}{*}{ Ln migration-weighted OECD price ${ }_{t-1}$} & & -0.077 & & -0.016 \\
\hline & & $(0.071)$ & & $(0.074)$ \\
\hline \multirow[t]{2}{*}{ Ln (exports/GDP) $)_{\mathrm{t}-1} *$ Share of capital-intensive exports } & 0.37 & 0.147 & & \\
\hline & $(0.390)$ & $(0.789)$ & & \\
\hline \multirow[t]{2}{*}{ Ln (imports/GDP) $)_{t-1} *$ Share of capital-intensive imports } & $1.335 * * *$ & $0.907 * *$ & & \\
\hline & $(0.416)$ & $(0.453)$ & & \\
\hline \multirow[t]{2}{*}{ Share of intermediates in overall imports } & & & $-3.871 *$ & -8.421 \\
\hline & & & $(2.154)$ & $(5.720)$ \\
\hline \multirow[t]{2}{*}{$\begin{array}{l}\text { Ln (imports/GDP) })_{\mathrm{t}-1} * \text { Share of intermediates in overall } \\
\text { imports }\end{array}$} & & & $1.072 *$ & 2.149 \\
\hline & & & $(0.647)$ & $(1.540)$ \\
\hline Country fixed effects & $\mathrm{Y}$ & $\mathrm{Y}$ & $\mathrm{Y}$ & $\mathrm{Y}$ \\
\hline Year fixed effects & $\mathrm{Y}$ & $\mathrm{Y}$ & $\mathrm{Y}$ & $\mathrm{Y}$ \\
\hline Observations & 740 & 378 & 729 & 378 \\
\hline Number of countries & 66 & 44 & 63 & 44 \\
\hline
\end{tabular}


Table A13. Effect of Exchange Rates on Wages-Interaction of Labor-DD With Labor Market Integration

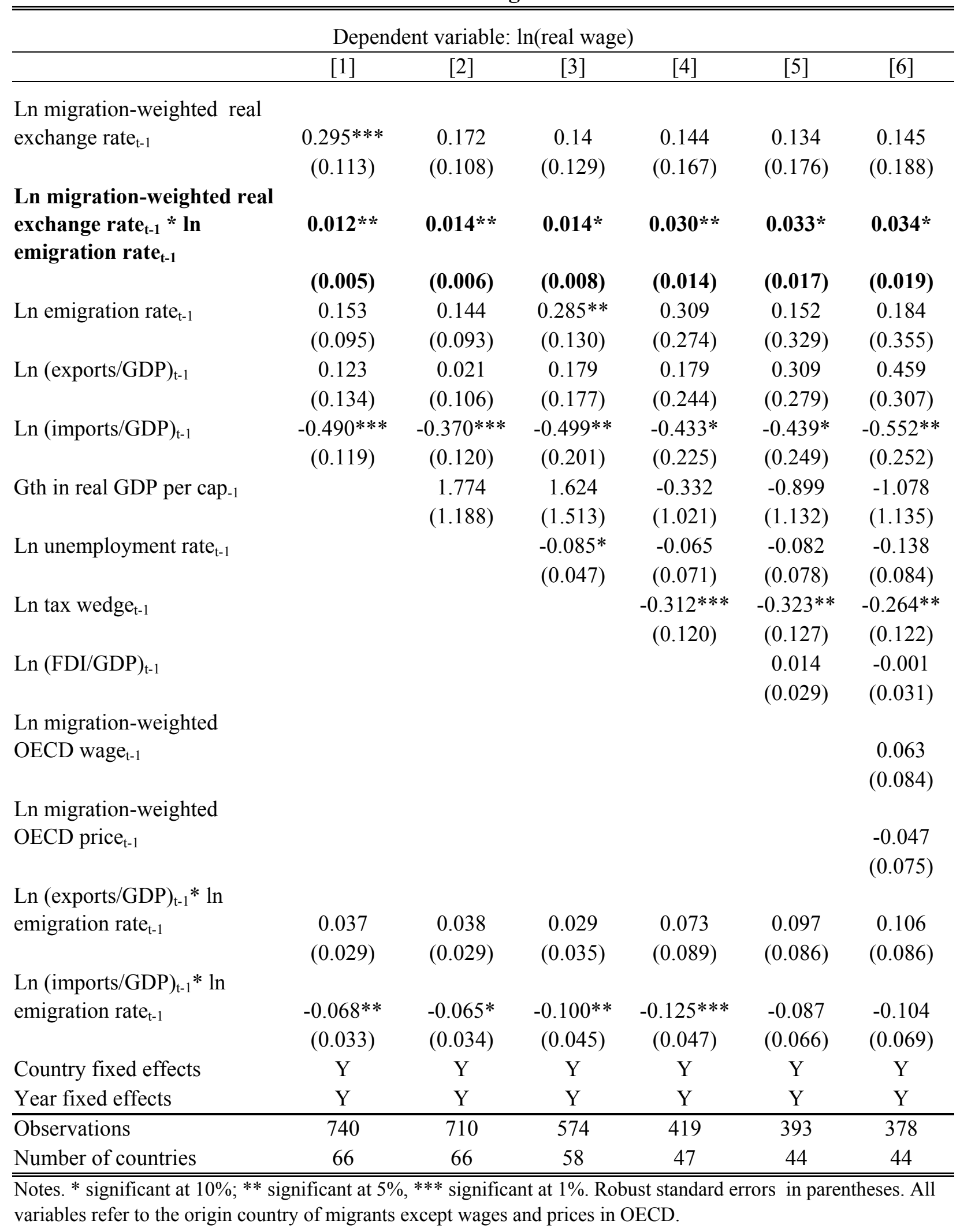


Table A14. List of Occupations: Freeman-Oostendorp Occupational Wages Around the World Database

\begin{tabular}{|c|c|c|c|c|c|}
\hline Occupation & Skill & Occupation & Skill & Occupation & Skill \\
\hline Farm supervisor & Skilled & Mixing- and blending-machine operator & Unskilled & Bus conductor & Unskilled \\
\hline Field crop farm worker & Unskilled & Labourer & Unskilled & Automobile mechanic & Unskilled \\
\hline Plantation supervisor & Skilled & Mixing- and blending-machine operator & Unskilled & Motor bus driver & Unskilled \\
\hline Plantation worker & Unskilled & Packer & Unskilled & Urban motor truck driver & Unskilled \\
\hline Forest supervisor & Skilled & Labourer & Unskilled & Long-distance motor truck driver & Unskilled \\
\hline Forestry worker & Unskilled & Controlman & Unskilled & Ship's chief engineer & Skilled \\
\hline Logger & Unskilled & Occupational health nurse & Skilled & Ship's steward (passenger) & Unskilled \\
\hline Tree feller and bucker & Unskilled & Blast furnaceman (ore smelting) & Unskilled & Able seaman & Unskilled \\
\hline Deep-sea fisherman & Unskilled & Hot-roller (steel) & Unskilled & Dock worker & Unskilled \\
\hline Inshore (coastal) maritime fisherman & Unskilled & Metal melter & Unskilled & Air transport pilot & Skilled \\
\hline Coalmining engineer & Skilled & Labourer & Unskilled & Flight operations officer & Skilled \\
\hline Miner & Skilled & Metalworking machine setter & Unskilled & Airline ground receptionist & Skilled \\
\hline Underground helper, loader & Unskilled & Welder & Unskilled & Aircraft cabin attendant & Skilled \\
\hline Petroleum and natural gas engineer & Skilled & Bench moulder (metal) & Unskilled & Aircraft engine mechanic & Unskilled \\
\hline $\begin{array}{l}\text { Petroleum and natural gas extraction } \\
\text { technician }\end{array}$ & Skilled & Machinery fitter-assembler & Unskilled & Aircraft loader & Unskilled \\
\hline Supervisor or general foreman & Skilled & Labourer & Unskilled & Air traffic controller & Skilled \\
\hline Derrickman & Unskilled & Electronics draughtsman & Unskilled & Aircraft accident fire-fighter & Skilled \\
\hline Miner & Skilled & Electronics engineering technician & Unskilled & Post office counter clerk & Skilled \\
\hline Quarryman & Unskilled & Electronics fitter & Unskilled & Postman & Skilled \\
\hline Butcher & Unskilled & Electronic equipment assembler & Unskilled & Telephone switchboard operator & Skilled \\
\hline Packer & Unskilled & Ship plater & Unskilled & Accountant & Skilled \\
\hline Dairy product processor & Unskilled & $\begin{array}{l}\text { Power distribution and transmission } \\
\text { engineer }\end{array}$ & Skilled & Stenographer-typist & Skilled \\
\hline Grain miller & Unskilled & Office clerk & Skilled & Bank teller & Skilled \\
\hline Baker (ovenman) & Unskilled & Electric power lineman & Unskilled & Book-keeping machine operator & Skilled \\
\hline Thread and yarn spinner & Unskilled & Power-generating machinery operator & Unskilled & Computer programmer & Skilled \\
\hline
\end{tabular}


Loom fixer, tuner

Cloth weaver (machine)

Labourer

Garment cutter

Sewing-machine operator

Tanner

Leather goods maker

Clicker cutter (machine)

Laster

Shoe sewer (machine)

Sawmill sawyer

Veneer cutter

Plywood press operator

Furniture upholsterer

Cabinetmaker

Wooden furniture finisher

Wood grinder

Paper-making-machine operator (wet end) Unskilled Hotel receptionist

Journalist

Stenographer-typist

Office clerk

Hand compositor

Machine compositor

Printing pressman

Bookbinder (machine)

Labourer

Chemical engineer
Unskilled Labourer

Unskilled Building electrician

Unskilled Plumber

Unskilled Constructional steel erector

Unskilled Building painter

Unskilled Bricklayer (construction)

Unskilled Reinforced concreter

Unskilled Cement finisher

Unskilled Construction carpenter

Unskilled Plasterer

Unskilled Labourer

Unskilled Stenographer-typist

Unskilled Stock records clerk

Unskilled Salesperson

Unskilled Book-keeper

Unskilled Cash desk cashier

Unskilled Salesperson

Skilled Cook

Skilled Waiter

Skilled Room attendant or chambermaid

Skilled Ticket seller (cash desk cashier)

Skilled Railway services supervisor

Skilled Railway passenger train guard

Skilled Railway vehicle loader

Unskilled Railway engine-driver

Skilled Railway steam-engine fireman
Unskilled Stenographer-typist

Skilled

Unskilled Card- and tape-punching-machine Skilled operator

Unskilled Insurance agent Skilled

Unskilled Clerk of works Skilled

Unskilled Computer programmer Skilled

Unskilled Government executive official: Skilled

Unskilled Stenographer-typist Skilled

Unskilled Card- and tape-punching- machine Skilled operator

Unskilled Office clerk Skilled

Unskilled Fire-fighter Skilled

Unskilled Refuse collector Unskilled

Skilled Mathematics teacher (third level) Skilled

Skilled Teacher in languages and literature (third Skilled level)

Skilled Teacher in languages and literature Skilled (second level)

Skilled Mathematics teacher (second level) Skilled

Skilled Technical education teacher (second level) Skilled

Skilled First-level education teacher Skilled

Skilled Kindergarten teacher Skilled

Unskilled General physician Skilled

Unskilled Dentist (general) Skilled

Unskilled Professional nurse (general) Skilled

Skilled Auxiliary nurse Skilled

Skilled Physiotherapist Skilled

Unskilled Medical X-ray technician Skilled

Unskilled Ambulance driver Unskilled

Unskilled Automobile mechanic Unskilled

Unskilled Pattern makers (wood) Unskilled 


\begin{tabular}{llll} 
Chemistry technician & Skilled & Railway signalman & Unskilled Permanent way labourers \\
Supervisor or general foreman & Skilled & Road transport services supervisor & $\begin{array}{l}\text { Skilled } \\
\text { Labourers (unskilled, public parks and } \\
\text { gardens) }\end{array}$ \\
\hline \hline
\end{tabular}

Source: http://www.nber.org/oww/ 
Table A15. Effect of Exchange Rates on Wages-Low and High Skill Wages

\begin{tabular}{|c|c|c|c|c|}
\hline \multicolumn{5}{|c|}{ Dependent variable } \\
\hline & $\ln ($ low-skill real wage $)$ & ln(high-skill real wage) & $\ln$ (low-skill real wage) & ln(high-skill real \\
\hline Ln migration-weighted real exchange rate $_{t-1}$ & $\begin{array}{c}3.880 * * * \\
(1.099)\end{array}$ & $\begin{array}{c}3.923 * * * \\
(1.043)\end{array}$ & $\begin{array}{c}0.182 \\
(0.128)\end{array}$ & $\begin{array}{l}-0.395 \\
(0.405)\end{array}$ \\
\hline $\begin{array}{l}\text { Ln migration-weighted real exchange } \\
\text { rate }_{t-1} * \text { Ln emigration rate } \\
t-1\end{array}$ & $\begin{array}{l}0.579 * * * \\
(0.062)\end{array}$ & $\begin{array}{l}0.577 * * * \\
(\mathbf{0 . 0 5 8})\end{array}$ & & \\
\hline $\begin{array}{l}\text { Ln migration-weighted real exchange } \\
\text { rate }_{t-1} * \text { Ln low-skill emigration rate } \\
t-1\end{array}$ & & & $\begin{array}{l}0.237 * * \\
(0.107)\end{array}$ & \\
\hline $\begin{array}{l}\text { Ln migration-weighted real exchange } \\
\text { rate }_{t-1} * \text { Ln high-skill emigration rate } \\
t-1\end{array}$ & & & & $\begin{array}{l}0.362 * * \\
(0.176)\end{array}$ \\
\hline Ln emigration rate $_{t-1}$ & $\begin{array}{l}1.122 * * * \\
(0.164)\end{array}$ & $\begin{array}{c}1.107 * * * \\
(0.156)\end{array}$ & & \\
\hline Ln low-skill emigration rate $\mathrm{t}_{\mathrm{t}-1}$ & & & $\begin{array}{c}0.212 \\
(0.243)\end{array}$ & \\
\hline Ln high-skill emigration rate $\mathrm{t}_{\mathrm{t}-1}$ & & & & $\begin{array}{c}1.326 \\
(0.882)\end{array}$ \\
\hline $\operatorname{Ln}(\text { exports/GDP })_{t-1}$ & $\begin{array}{c}0.089 \\
(0.800)\end{array}$ & $\begin{array}{l}-0.054 \\
(0.755)\end{array}$ & $\begin{array}{c}1.022 \\
(0.836)\end{array}$ & $\begin{array}{c}1.018 \\
(1.072)\end{array}$ \\
\hline Ln (imports/GDP) $)_{\mathrm{t}-1}$ & $\begin{array}{l}-3.930 * * * \\
(1.366)\end{array}$ & $\begin{array}{l}-3.810^{* * *} \\
(1.269)\end{array}$ & $\begin{array}{c}-0.1 \\
(0.772)\end{array}$ & $\begin{array}{l}-0.957 \\
(1.235)\end{array}$ \\
\hline Growth in real GDP per capita ${ }_{t-1}$ & $\begin{array}{l}-1.709 \\
(3.857)\end{array}$ & $\begin{array}{l}-1.631 \\
(3.729)\end{array}$ & $\begin{array}{l}-3.539 \\
9.543)\end{array}$ & $\begin{array}{c}-1.594 \\
(10.032)\end{array}$ \\
\hline Ln unemployment rate ${ }_{t-1}$ & $\begin{array}{l}-0.42 \\
(0.296)\end{array}$ & $\begin{array}{c}-0.464 * \\
(0.277)\end{array}$ & $\begin{array}{c}-0.32 \\
(0.380)\end{array}$ & $\begin{array}{l}-0.633 \\
(0.426)\end{array}$ \\
\hline Ln tax wedge $\mathrm{t}_{\mathrm{t}-1}$ & $\begin{array}{c}1.565 \\
(1.279)\end{array}$ & $\begin{array}{c}1.441 \\
(1.183)\end{array}$ & $\begin{array}{l}-0.085 \\
(0.586)\end{array}$ & $\begin{array}{l}-0.427 \\
(0.563)\end{array}$ \\
\hline
\end{tabular}




\begin{tabular}{|c|c|c|c|c|}
\hline $\operatorname{Ln}(\mathrm{FDI} / \mathrm{GDP})_{\mathrm{t}-1}$ & $\begin{array}{c}-0.304^{* * * *} \\
(0.093)\end{array}$ & $\begin{array}{c}-0.270^{* * * *} \\
(0.088)\end{array}$ & $\begin{array}{c}-0.784 * * * \\
(0.255)\end{array}$ & $\begin{array}{c}-0.875^{* * *} \\
(0.278)\end{array}$ \\
\hline Ln migration-weighted OECD wage $_{t-1}$ & $\begin{array}{c}-0.560^{*} \\
(0.324)\end{array}$ & $\begin{array}{c}-0.510^{*} \\
(0.305)\end{array}$ & $\begin{array}{c}0.228 * * * \\
(0.086)\end{array}$ & $\begin{array}{c}0.477 * * * \\
(0.098)\end{array}$ \\
\hline Ln migration-weighted OECD price $_{t-1}$ & $\begin{array}{c}0.514 \\
(1.504)\end{array}$ & $\begin{array}{c}0.264 \\
(1.384)\end{array}$ & $\begin{array}{c}3.597 \\
(2.200)\end{array}$ & $\begin{array}{c}3.029 \\
(2.088)\end{array}$ \\
\hline Country fixed effects & $\mathrm{Y}$ & $\mathrm{Y}$ & $\mathrm{N}$ & $\mathrm{N}$ \\
\hline Year fixed effects & $\mathrm{Y}$ & $\mathrm{Y}$ & $\mathrm{Y}$ & $\mathrm{Y}$ \\
\hline Observations & 151 & 151 & 151 & 151 \\
\hline Number of countries & 30 & 30 & 30 & 30 \\
\hline
\end{tabular}

Notes. * significant at $10 \%$; ${ }^{* *}$ significant at $5 \%,{ }^{* * *}$ significant at $1 \%$. Robust standard errors in parentheses. All variables refer to the origin country of migrants except wages and prices in OECD. 
Table A16. Effect of Exchange Rates on Wages-Interaction With Labor Market Integration: Split Samples Pre and Post 1993

\begin{tabular}{|c|c|c|}
\hline \multicolumn{3}{|l|}{ Dependent variable: $\ln ($ real wage $)$} \\
\hline & $\begin{array}{c}\text { Pre-1993 } \\
{[1]}\end{array}$ & $\begin{array}{c}\text { Post } 1993 \\
{[2]}\end{array}$ \\
\hline Ln migration-weighted real exchange rate $_{t-1}$ & $\begin{array}{c}0.136 \\
(0.104)\end{array}$ & $\begin{array}{c}0.136 \\
(0.292)\end{array}$ \\
\hline Ln migration-weighted real exchange rate $_{t-1} *$ In emigration rate $_{t-1}$ & $\begin{array}{l}0.013 * \\
(0.008)\end{array}$ & $\begin{array}{c}\mathbf{0 . 1 2} * * * \\
(0.045)\end{array}$ \\
\hline Ln emigration rate $_{t-1}$ & $\begin{array}{c}0.082 * * \\
(0.075)\end{array}$ & $\begin{array}{c}0.316^{* * *} \\
(0.101)\end{array}$ \\
\hline $\operatorname{Ln}(\text { exports/GDP })_{t-1}$ & $\begin{array}{c}-0.345^{* *} \\
(0.147)\end{array}$ & $\begin{array}{c}0.118 \\
(0.425)\end{array}$ \\
\hline Ln (imports/GDP) $)_{\mathrm{t}-1}$ & $\begin{array}{c}0.226 \\
(0.216)\end{array}$ & $\begin{array}{c}-0.002 \\
(0.436)\end{array}$ \\
\hline Growth in real GDP per capita $\mathrm{t}_{-1}$ & $\begin{array}{c}0.163 \\
(0.681)\end{array}$ & $\begin{array}{c}0.009 \\
(1.047)\end{array}$ \\
\hline Ln unemployment rate $_{t-1}$ & $\begin{array}{c}0.102 * * * \\
(0.034)\end{array}$ & $\begin{array}{l}-0.159 \\
(0.125)\end{array}$ \\
\hline Ln tax wedge $\mathrm{t}_{\mathrm{t}-1}$ & $\begin{array}{l}-0.15^{*} \\
(0.075)\end{array}$ & $\begin{array}{c}-0.118 \\
(0.187)\end{array}$ \\
\hline $\operatorname{Ln}(\mathrm{FDI} / \mathrm{GDP})_{\mathrm{t}-1}$ & $\begin{array}{c}-0.008 \\
(0.023)\end{array}$ & $\begin{array}{c}0.034 \\
(0.035)\end{array}$ \\
\hline Ln migration-weighted OECD wage $\mathrm{t}_{-1}$ & $\begin{array}{c}0.137 \\
(0.111)\end{array}$ & $\begin{array}{c}0.082 \\
(0.094)\end{array}$ \\
\hline Ln migration-weighted OECD price t-1 $_{1}$ & $\begin{array}{c}0.065 \\
(0.047)\end{array}$ & $\begin{array}{l}-0.089 \\
(0.107)\end{array}$ \\
\hline Country fixed effects & Y & Y \\
\hline Year fixed effects & $\mathrm{Y}$ & $\mathrm{Y}$ \\
\hline Observations & 110 & 268 \\
\hline Number of countries & 22 & 17 \\
\hline
\end{tabular}


Table A17. Effect of Exchange Rates on Wages-Interaction With Labor Market Integration: Split Samples by Remittances

\begin{tabular}{|c|c|c|}
\hline \multicolumn{3}{|c|}{ Dependent variable: $\ln ($ real wage) } \\
\hline & $\begin{array}{l}\text { High remittance } \\
\qquad[1]\end{array}$ & $\begin{array}{c}\text { Low remittance } \\
{[2]}\end{array}$ \\
\hline Ln migration-weighted real exchange rate ${ }_{t-1}$ & $\begin{array}{c}0.551 \\
(0.339)\end{array}$ & $\begin{array}{c}0.105 \\
(0.248)\end{array}$ \\
\hline $\begin{array}{l}\text { Ln migration-weighted real exchange } \\
\text { rate }_{t-1} * \text { In emigration rate } \text { t }-1\end{array}$ & $\begin{array}{l}0.075 * \\
(0.038)\end{array}$ & $\begin{array}{l}-0.187 \\
(0.116)\end{array}$ \\
\hline Ln emigration rate t $1_{1}$ & $\begin{array}{l}0.175 * * \\
(0.078)\end{array}$ & $\begin{array}{l}-0.375 \\
(0.509)\end{array}$ \\
\hline $\operatorname{Ln}(\text { exports/GDP })_{\mathrm{t}-1}$ & $\begin{array}{c}0.011 \\
(0.327)\end{array}$ & $\begin{array}{c}-0.34 \\
(0.504)\end{array}$ \\
\hline Ln (imports/GDP) $)_{\mathrm{t}-1}$ & $\begin{array}{l}-0.588 \\
(0.411)\end{array}$ & $\begin{array}{c}0.793 \\
(0.484)\end{array}$ \\
\hline Growth in real GDP per capita ${ }_{t-1}$ & $\begin{array}{l}-2.279 \\
(1.461)\end{array}$ & $\begin{array}{c}0.584 \\
(1.021)\end{array}$ \\
\hline Ln unemployment rate $_{t-1}$ & $\begin{array}{l}-0.289 \\
(0.197)\end{array}$ & $\begin{array}{c}0.117 \\
(0.108)\end{array}$ \\
\hline Ln tax wedge $\mathrm{t}_{\mathrm{t}-1}$ & $\begin{array}{c}-0.06 \\
(0.299)\end{array}$ & $\begin{array}{l}-0.118 \\
(0.229)\end{array}$ \\
\hline $\operatorname{Ln}(\mathrm{FDI} / \mathrm{GDP})_{\mathrm{t}-1}$ & $\begin{array}{l}-0.028 \\
(0.064)\end{array}$ & $\begin{array}{c}0.012 \\
(0.038)\end{array}$ \\
\hline Ln migration-weighted OECD wage $_{t-1}$ & $\begin{array}{l}-0.013 \\
(0.171)\end{array}$ & $\begin{array}{l}-0.164 * \\
(0.091)\end{array}$ \\
\hline Ln migration-weighted OECD price t- $_{1}$ & $\begin{array}{l}-0.111 \\
(0.180)\end{array}$ & $\begin{array}{c}0.247 \\
(0.219)\end{array}$ \\
\hline Country fixed effects & $\mathrm{Y}$ & $\mathrm{Y}$ \\
\hline Year fixed effects & Y & Y \\
\hline Observations & 157 & 153 \\
\hline Number of countries & 22 & 17 \\
\hline
\end{tabular}

The regression sample corresponding to Table 2, column [6] are split by remittances. Countries with high and low trend $(\log )$ remittances are defined by those above and below the median. ${ }^{*}$ significant at $10 \%$; $* *$ significant at $5 \%, * * *$ significant at $1 \%$. Robust standard errors in parentheses. All variables refer to the origin country of migrants except wages and prices in OECD. 